\title{
On Generalized Fibonacci Numbers
}

\author{
Fidel Ochieng Oduol ${ }^{1}$, Isaac Owino Okoth ${ }^{2 *}$
}

\begin{abstract}
Fibonacci numbers and their polynomials have been generalized mainly by two ways: by maintaining the recurrence relation and varying the initial conditions, and by varying the recurrence relation and maintaining the initial conditions. In this paper, we introduce and derive various properties of $r$-sum Fibonacci numbers. The recurrence relation is maintained but initial conditions are varied. Among results obtained are Binet's formula, generating function, explicit sum formula, sum of first $n$ terms, sum of first $n$ terms with even indices, sum of first $n$ terms with odd indices, alternating sum of $n$ terms of $r$-sum Fibonacci sequence, Honsberger's identity, determinant identities and a generalized identity from which Cassini's identity, Catalan's identity and d'Ocagne's identity follow immediately.
\end{abstract}

Keywords: Binet's formula, Fibonacci sequence, generating function, $r$-sum Fibonacci sequence 2010 AMS: 11B39

${ }^{1}$ Department of Pure and Applied Mathematics, Maseno University, Maseno, Kenya, ORCID: 0000-0002-1228-6339

${ }^{2}$ Department of Pure and Applied Mathematics, Maseno University, Maseno, Kenya, ORCID: 0000-0003-4503-4733

*Corresponding author: ookoth@maseno.ac.ke

Received: 17 July 2020, Accepted: 29 September 2020, Available online: 22 December 2020

\section{Introduction}

Fibonacci sequence is the most studied sequence in the history of mathematics. In [14], the said sequence is given by A000045. The sequence is generated by a recursive formula $f_{n}=f_{n-1}+f_{n-2}$, for $n \geq 3$ with $f_{1}=0$ and $f_{2}=1$. The sequence has many interesting properties. For example, the ratio $\frac{f_{n+1}}{f_{n}}$ converges to the golden ratio $\frac{1+\sqrt{5}}{2}$ as $n$ tends to infinity.

Various generalizations of the aforementioned sequence have been derived since it was first discovered by Fibonacci in the $13^{\text {th }}$ century. Fibonacci sequence has been generalized mainly by two ways: by maintaining the recurrence relation and varying the initial conditions $[1,3,4,5,7,9,10]$, and by varying the recurrence relation and maintaining the initial conditions $[2,4,8,9,11,13,12,15]$. Some of the properties that have been obtained by various researchers are not limited to finding a closed form for the $n^{\text {th }}$ term of the sequence, sum of the first $n$ terms of the sequence, sum of the first $n$ terms with odd (or even) indices of the sequence, explicit sum formula, Catalan's identity, Cassini's identity, d'Ocagne's identity, Honsberger's identity, determinant identities, and generating function among many others.

Let $f_{n}$ be the $n^{\text {th }}$ term of Fibonacci sequence. Binet's formula gives a closed formula for $f_{n}$ as

$$
f_{n}=\frac{1}{\alpha-\beta}\left(\alpha^{n-1}-\beta^{n-1}\right),
$$

where $\alpha=\frac{1+\sqrt{5}}{2}$ and $\beta=\frac{1-\sqrt{5}}{2}$.

Companion to Fibonacci numbers are Lucas numbers with the same recurrence relation as Fibonacci numbers except for 
initial conditions which are 2 and 1 . Binet's formula for Lucas numbers, $l_{n}$, is given by

$$
l_{n}=\alpha^{n-1}+\beta^{n-1} .
$$

Here, $l_{n}$ is the $n^{\text {th }}$ Lucas number.

Some properties of Fibonacci sequences explored in this paper include the sum of the first $n$ terms of Fibonacci sequence,

$$
f_{1}+f_{2}+f_{3}+\cdots+f_{n}=f_{n+2}-1,
$$

and the Honsberger's identity

$$
f_{n+m}=f_{n} f_{m}+f_{n+1} f_{m+1}
$$

for all $n \geq 1$ and $n>m$.

Definition 1.1. The $n^{\text {th }}$ term of $r$-sum Fibonacci sequence, $h_{n, r}$, is given by

$$
h_{n, r}=f_{n}+f_{n+1}+\cdots+f_{n+r-1} .
$$

Using Definition 1.1, it follows that the first term

$$
h_{1, r}=f_{1}+f_{2}+\cdots+f_{r}=f_{r+2}-1
$$

and the second term

$$
h_{2, r}=f_{2}+f_{3}+\cdots+f_{r+1}=f_{r+3}-1 .
$$

As with Fibonacci sequence, the $r$-sum Fibonacci sequence satisfies the recurrence relation

$$
h_{n, r}=h_{n-1, r}+h_{n-2, r},
$$

for $n \geq 3$, with initial conditions $h_{1, r}=f_{r+2}-1$ and $h_{2, r}=f_{r+3}-1$.

Few entries of $h_{n, r}$ are given in Table 1 below.

Table 1. $r$-Sum Fibonacci numbers

\begin{tabular}{|c|c|c|c|c|c|c|c|c|c|c|c|}
\hline$r$ & $h_{1, r}$ & $h_{2, r}$ & $h_{3, r}$ & $h_{4, r}$ & $h_{5, r}$ & $h_{6, r}$ & $h_{7, r}$ & $h_{8, r}$ & $h_{9, r}$ & $h_{10, r}$ & $h_{11, r}$ \\
\hline 1 & 0 & 1 & 1 & 2 & 3 & 5 & 8 & 13 & 21 & 34 & 55 \\
2 & 1 & 2 & 3 & 5 & 8 & 13 & 21 & 34 & 55 & 89 & 144 \\
3 & 2 & 4 & 6 & 10 & 16 & 26 & 42 & 68 & 110 & 178 & 288 \\
4 & 4 & 7 & 11 & 18 & 29 & 47 & 76 & 123 & 199 & 322 & 521 \\
5 & 7 & 12 & 19 & 31 & 50 & 81 & 131 & 212 & 343 & 555 & 898 \\
6 & 12 & 20 & 32 & 52 & 84 & 136 & 220 & 356 & 576 & 932 & 1508 \\
7 & 20 & 33 & 53 & 86 & 139 & 225 & 364 & 589 & 953 & 1542 & 2495 \\
\hline
\end{tabular}

When $r=1,2$, we get Fibonacci sequence with different initial conditions. For $r \geq 3$, we get Fibonacci-like numbers. We also note that when $r=4$, we obtain Lucas numbers.

This paper is organized as follows: Some basic properties of $h_{n, r}$ are given in Section 2. In Section 3, we obtain Binet's formula and generating function for these numbers. Further properties of these numbers are presented in Section 4. Moreover, determinant identities are presented in Section 5. We conclude the paper in Section 6.

\section{Preliminary Results}

We start off, with these important and interesting properties:

Lemma 2.1. For $n \geq 1$, we have $h_{n, 3}=2 h_{n, 2}$. 
Proof. From (1.5), we have

$$
\begin{aligned}
h_{n, 3} & =f_{n}+f_{n+1}+f_{n+2}, \\
& =f_{n}+f_{n+1}+f_{n}+f_{n+1}, \\
& =2\left(f_{n}+f_{n+1}\right), \\
& =2 h_{n, 2} .
\end{aligned}
$$

Proposition 2.2. The $n^{\text {th }}$ term of $r$-sum Fibonacci number, $h_{n, r}$, can be expressed as $h_{n, r}=f_{n+r+1}-f_{n+1}$, for all $r \geq 1$.

Proof. From recurrence relation (1.6) and equation (1.5), we have

$$
\begin{aligned}
h_{n, r} & =h_{n-1, r}+h_{n-2, r}, \\
& =\left(f_{n-1}+f_{n}+\cdots+f_{n+r-2}\right)+\left(f_{n-2}+f_{n-1}+\cdots+f_{n+r-3}\right), \\
& =\left[\left(f_{1}+f_{2}+\cdots+f_{n+r-2}-\left(f_{1}+f_{2}+\cdots+f_{n-2}\right)\right]+\left[\left(f_{1}+f_{2}+\cdots+f_{n+r-3}\right)-\left(f_{1}+f_{2}+\cdots+f_{n-3}\right)\right] .\right.
\end{aligned}
$$

By equation (1.3), we get

$$
\begin{aligned}
h_{n, r} & =\left[\left(f_{n+r}-1\right)-\left(f_{n}-1\right)\right]+\left[\left(f_{n+r-1}-1\right)-\left(f_{n-1}-1\right)\right] \\
& =f_{n+r}-f_{n}+f_{n+r-1}-f_{n-1} \\
& =f_{n+r+1}-f_{n+1} .
\end{aligned}
$$

\section{Proposition 2.3.}

$$
h_{n, r}=f_{r+1} f_{n+2}+f_{n+1} \sum_{i=1}^{r-2} f_{i} .
$$

Proof. By Proposition 2.2, we have that

$$
h_{n, r}=f_{n+r+1}-f_{n+1} \text {. }
$$

Now, by Honsberger's identity (1.4), we have

$$
f_{n+r+1}=f_{r+1} f_{n+2}+f_{r} f_{n+1} .
$$

Substituting this sum in (2.1), we obtain

$$
\begin{aligned}
h_{n, r} & =f_{r+1} f_{n+2}+f_{r} f_{n+1}-f_{n+1} \\
& =f_{r} f_{n+2}+f_{n+1}\left(f_{r}-1\right) .
\end{aligned}
$$

Since $\sum_{i=1}^{n} f_{i}=f_{n+2}-1$, then $h_{n, r}=f_{r+1} f_{n+2}+f_{n+1} \sum_{i=1}^{r-2} f_{i}$.

Theorem 2.4. The numbers, $h_{n, r}$, can be expressed in terms of Fibonacci and Lucas numbers as:

$$
h_{n, r}=\left\{\begin{array}{llr}
\sum_{i=1}^{m} l_{n+4 i-1} & \text { if } \quad r=4 m, \\
\sum_{i=1}^{m} l_{n+4 i-1}+f_{n+4 m} & \text { if } & r=4 m+1, \\
\sum_{i=1}^{m} l_{n+4 i-1}+f_{n+4 m+2} & \text { if } & r=4 m+2, \\
\sum_{i=1}^{m} l_{n+4 i-1}+2 f_{n+4 m+2} & \text { if } & r=4 m+3 .
\end{array}\right.
$$


Proof. If $r=4 m$, then

$$
\begin{aligned}
h_{n, r} & =f_{n}+f_{n+1}+\cdots+f_{n+4 m-1} \\
& =f_{n+2}+f_{n+4}+\cdots+f_{n+4 m} \\
& =l_{n+3}+l_{n+7}+\cdots+l_{n+4 m-1} .
\end{aligned}
$$

If $r=4 m+1$, then

$$
\begin{aligned}
h_{n, r} & =f_{n}+f_{n+1}+\cdots+f_{n+4 m} \\
& =f_{n+2}+f_{n+4}+\cdots+f_{n+4 m-2}+f_{n+4 m}+f_{n+4 m} \\
& =l_{n+3}+l_{n+7}+\cdots+l_{n+4 m-1}+f_{n+4 m} .
\end{aligned}
$$

If $r=4 m+2$, then

$$
\begin{aligned}
h_{n, r} & =f_{n}+f_{n+1}+f_{n+2}+\cdots+f_{n+4 m+1} \\
& =f_{n+2}+f_{n+4}+\cdots+f_{n+4 m+2} \\
& =l_{n+3}+l_{n+7}+\cdots+l_{n+4 m-1}+f_{n+4 m+2} .
\end{aligned}
$$

If $r=4 m+3$, then

$$
\begin{aligned}
h_{n, r} & =f_{n}+f_{n+1}+\cdots+f_{n+4 m+2} \\
& =f_{n+2}+f_{n+4}+\cdots+f_{n+4 m}+f_{n+4 m+2}+f_{n+4 m+2} \\
& =l_{n+3}+l_{n+7}+\cdots+l_{n+4 m-1}+2 f_{n+4 m+2} .
\end{aligned}
$$

Remark 2.5. We note that:

1. For $r=1,2$, the $r$-sum Fibonacci numbers, $h_{n, r}$, are themselves Fibonacci numbers.

2. We have $h_{n, 3}$ as a sum of Fibonacci numbers for all $n \geq 1$.

3. The numbers, $h_{n, 4}$, are Lucas numbers for all integers $n \geq 1$.

4. The numbers, $h_{n, 4 m}$, are sums of Lucas numbers for all integers $m \geq 1$ and $n \geq 1$.

5. For all $m \in \mathbb{N}$ and $n \geq 1$, we have that $h_{n, 4 m+1}, h_{n, 4 m+2}$, and $h_{n, 4 m+3}$ are sums of Fibonacci and Lucas numbers.

Proposition 2.6. Let $m \geq 1$. Then the $n^{\text {th }}$ term of $4 m$-sum Fibonacci sequence, $h_{n, 4 m}$, satisfies the equation

$$
h_{n, 4 m}=f_{2 m+1} l_{n+2 m+1} .
$$

Proof. By Binet's formulas for Fibonacci numbers (1.1) and Lucas numbers (1.2) and by equation (2.1), we have

$$
\begin{aligned}
h_{n, 4 m} & =f_{n+4 m+1}-f_{n+1} \\
& =\frac{1}{\alpha-\beta}\left(\alpha^{n+4 m}-\beta^{n+4 m}\right)-\frac{1}{\alpha-\beta}\left(\alpha^{n}-\beta^{n}\right) .
\end{aligned}
$$

Since $\alpha \beta=-1$ then, $(\alpha \beta)^{2 m}=1$, and

$$
\begin{aligned}
h_{n, 4 m} & =\frac{1}{\alpha-\beta}\left(\alpha^{n+4 m}-(\alpha \beta)^{2 m} \alpha^{n}-\beta^{n+4 m}+(\alpha \beta)^{2 m} \beta^{n}\right) \\
& =\frac{1}{\alpha-\beta}\left(\alpha^{n+4 m}-\beta^{2 m} \alpha^{n+2 m}-\beta^{n+4 m}+\alpha^{2 m} \beta^{n+2 m}\right) \\
& =\frac{1}{\alpha-\beta}\left(\alpha^{n+2 m}\left(\alpha^{2 m}-\beta^{2 m}\right)+\beta^{n+2 m}\left(\alpha^{2 m}-\beta^{2 m}\right)\right) \\
& =\frac{1}{\alpha-\beta}\left(\alpha^{2 m}-\beta^{2 m}\right)\left(\alpha^{n+2 m}+\beta^{n+2 m}\right) \\
& =f_{2 m+1} l_{n+2 m+1} .
\end{aligned}
$$


Setting $m=1$ in Proposition 2.6, we get:

Corollary 2.7. $h_{n, 4}=l_{n+3}$, for all $n \geq 1$.

Proposition 2.8.

$$
\sum_{k=2}^{n} h_{k, r}^{2}=h_{n, r} h_{n+1, r}-h_{1, r} h_{2, r}
$$

Proof. Since $h_{n, r}=h_{n+1, r}-h_{n-1, r}$ then,

$$
h_{n, r}^{2}=h_{n, r} h_{n+1, r}-h_{n-1, r} h_{n, r} .
$$

Now, we have

$$
\begin{aligned}
h_{2, r}^{2}= & h_{2, r} h_{3, r}-h_{1, r} h_{2, r} \\
h_{3, r}^{2}= & h_{3, r} h_{4, r}-h_{2, r} h_{3, r} \\
h_{4, r}^{2}= & h_{3, r} h_{5, r}-h_{3, r} h_{4, r} \\
& \vdots \\
h_{n-1, r}^{2}= & h_{n-1, r} h_{n, r}-h_{n-2, r} h_{n-1, r} \\
h_{n, r}^{2}= & h_{n, r} h_{n+1, r}-h_{n-1, r} h_{n, r} .
\end{aligned}
$$

Adding up these equations, we get

$$
h_{2, r}^{2}+h_{3, r}^{2}+h_{4, r}^{2}+\cdots+h_{n-1, r}^{2}+h_{n, r}^{2}=h_{n, r} h_{n+1}-h_{1, r} h_{2, r} \cdot
$$

Proposition 2.9. For every positive integer $n \geq 2$,

$$
h_{n, r}^{2}-h_{n-1, r}^{2}=h_{n+1, r} h_{n-2, r} .
$$

Proof. Since

$$
h_{n-1, r}^{2}=h_{n-1, r} h_{n, r}-h_{n-1, r} h_{n-2, r}
$$

then,

$$
\begin{aligned}
h_{n, r}^{2}-h_{n-1, r}^{2} & =h_{n, r}^{2}-h_{n-1, r} h_{n, r}+h_{n-1, r} h_{n-2, r} \\
& =h_{n, r}\left(h_{n, r}-h_{n-1, r}\right)+h_{n-1, r} h_{n-2, r} \\
& =h_{n, r} h_{n-2, r}+h_{n-1, r} h_{n-2, r} \\
& =h_{n-2, r}\left(h_{n, r}+h_{n-1, r}\right) \\
& =h_{n+1, r} h_{n-2, r} .
\end{aligned}
$$

\section{Binet's Formula and Generating Function}

We start by getting a closed formula for $h_{n, r}$.

Theorem 3.1 (Binet's Formula). The $n^{\text {th }}$ term of $r$-sum Fibonacci sequence, $h_{n, r}$, is given by

$$
h_{n, r}=\frac{1}{\alpha-\beta}\left[\left(h_{2, r}-\beta h_{1, r}\right) \alpha^{n-1}-\left(h_{2, r}-\alpha h_{1, r}\right) \beta^{n-1}\right] \text {, }
$$

where $\alpha=\frac{1+\sqrt{5}}{2}$ and $\beta=\frac{1-\sqrt{5}}{2}$. 
Proof. Let $n \geq 2$, then $r$-sum Fibonacci numbers are defined by the recurrence relation

$$
h_{n, r}=h_{n-1, r}+h_{n-2, r},
$$

with initial conditions $h_{1, r}=f_{r+2}-1$ and $h_{2, r}=f_{r+3}-1$, for all $r>0$. The characteristic equation of the recurrence relation is $\lambda^{2}-\lambda-1=0$. We solve this equation to get its roots as

$$
\alpha=\frac{1+\sqrt{5}}{2} \text { and } \beta=\frac{1-\sqrt{5}}{2} \text {. }
$$

These roots are real and distinct and thus the solution of the recurrence relation is of the form

$$
h_{n, r}=A \alpha^{n}+B \beta^{n},
$$

where $A$ and $B$ are constants.

Setting $n=1$ and $n=2$ in (3.2), we obtain

$$
A \alpha+B \beta=h_{1, r}
$$

and

$$
A \alpha^{2}+B \beta^{2}=h_{2, r}
$$

respectively. Solving these equations simultaneously, we get

$$
A=\frac{h_{2, r}-\beta h_{1, r}}{\alpha(\alpha-\beta)}
$$

and

$$
B=\frac{\alpha h_{1, r}-h_{2, r}}{\beta(\alpha-\beta)} .
$$

Thus the result follows.

Corollary 3.2. The $n^{\text {th }}$ term of the $r$-sum Fibonacci sequence satisfies the equation $h_{n, r}=h_{2, r} f_{n}+h_{1, r} f_{n-1}$.

Proof. From Binet's formula (3.1), we have

$$
h_{n, r}=\frac{1}{\alpha-\beta}\left[h_{2, r}\left(\alpha^{n-1}-\beta^{n-1}\right)-h_{1, r}(\alpha \beta)\left(\alpha^{n-2}-\beta^{n-2}\right)\right],
$$

where $\alpha=\frac{1+\sqrt{5}}{2}$ and $\beta=\frac{1-\sqrt{5}}{2}$. Since $\alpha \beta=-1$, then

$$
\begin{aligned}
h_{n, r} & =\frac{1}{\alpha-\beta}\left[h_{2, r}\left(\alpha^{n-1}-\beta^{n-1}\right)+h_{1, r}\left(\alpha^{n-2}-\beta^{n-2}\right)\right] \\
& =h_{2, r} f_{n}+h_{1, r} f_{n-1} .
\end{aligned}
$$

The following formula is rediscovered immediately upon setting $r=1$ in (3.1).

Corollary 3.3 (Binet's formula). The $n^{\text {th }}$ Fibonacci number, $f_{n}$, is given explicitly as

$$
f_{n}=\frac{1}{\alpha-\beta}\left[\alpha^{n-1}-\beta^{n-1}\right]
$$

where $\alpha=\frac{1+\sqrt{5}}{2}$ and $\beta=\frac{1-\sqrt{5}}{2}$. 
Corollary 3.4. The sequence of ratio of successive r-sum Fibonacci numbers $\frac{h_{n+1, r}}{h_{n, r}}$ converges to the golden ratio, i.e., $\lim _{n \rightarrow \infty} \frac{h_{n+1, r}}{h_{n, r}}=\frac{1+\sqrt{5}}{2}$.

Proof. From Binet's formula (3.1), we have

$$
\lim _{n \rightarrow \infty} \frac{h_{n+1, r}}{h_{n, r}}=\lim _{n \rightarrow \infty} \frac{\frac{1}{\alpha-\beta}\left[\left(h_{2, r}-\beta h_{1, r}\right) \alpha^{n}-\left(h_{2, r}-\alpha h_{1, r}\right) \beta^{n}\right]}{\frac{1}{\alpha-\beta}\left[\left(h_{2, r}-\beta h_{1, r}\right) \alpha^{n-1}-\left(h_{2, r}-\alpha h_{1, r}\right) \beta^{n-1}\right]},
$$

where $\alpha=\frac{1+\sqrt{5}}{2}$ and $\beta=\frac{1-\sqrt{5}}{2}$.

Factorizing $\alpha^{n-1}$, we obtain

$$
\lim _{n \rightarrow \infty} \frac{h_{n+1}, r}{h_{n, r}}=\lim _{n \rightarrow \infty} \frac{\frac{1}{\alpha-\beta} \alpha^{n-1}\left[\left(h_{2, r}-\beta h_{1, r}\right) \alpha-\left(h_{2, r}-\alpha h_{1, r}\right) \alpha^{-(n-1)} \beta^{n}\right]}{\frac{1}{\alpha-\beta} \alpha^{n-1}\left[\left(h_{2, r}-\beta h_{1, r}\right)-\left(h_{2, r}-\alpha h_{1, r}\right) \alpha^{-(n-1)} \beta^{n-1}\right]},
$$

which simplifies to

$$
\lim _{n \rightarrow \infty} \frac{h_{n+1, r}}{h_{n, r}}=\lim _{n \rightarrow \infty} \frac{\left(h_{2, r}-\beta h_{1, r}\right) \alpha-\left(h_{2, r}-\alpha h_{1, r}\right)\left(\frac{\beta}{\alpha}\right)^{n-1} \beta}{\left(h_{2, r}-\beta h_{1, r}\right)-\left(h_{2, r}-\alpha h_{1, r}\right)\left(\frac{\beta}{\alpha}\right)^{n-1}} .
$$

Since $\left|\frac{\beta}{\alpha}\right|<1$, we have $\lim _{n \rightarrow \infty}\left(\frac{\beta}{\alpha}\right)^{n-1}=0$ so that

$$
\lim _{n \rightarrow \infty} \frac{h_{n+1, r}}{h_{n, r}}=\lim _{n \rightarrow \infty} \frac{\left(h_{2, r}-\beta h_{1, r}\right) \alpha}{\left(h_{2, r}-\beta h_{1, r}\right)}=\alpha=\frac{1+\sqrt{5}}{2} .
$$

We now obtain the generating function for $r$-sum Fibonacci sequence.

Theorem 3.5. Let $H_{r}(t)$ be the generating function for $r$-sum Fibonacci sequence, then

$$
H_{r}(t)=\frac{h_{1, r} t+t^{2}\left(h_{2, r}-h_{1, r}\right)}{1-t-t^{2}}
$$

Proof. Let $H_{r}(t)=\sum_{n=1}^{\infty} h_{n, r} t^{n}$ be the generating function for $r$-sum Fibonacci numbers, then from $h_{n, r}=h_{n-1, r}+h_{n-2, r}$, we have

$$
\sum_{n \geq 3} h_{n, r} t^{n}=\sum_{n \geq 3} h_{n-1, r} t^{n}+\sum_{n \geq 3} h_{n-2, r} t^{n}
$$

This is the same as

$$
\sum_{n \geq 1} h_{n, r} t^{n}-h_{2, r} t^{2}-h_{1, r} t=t \sum_{n \geq 2} h_{n, r} t^{n}+t^{2} \sum_{n \geq 1} h_{n, r} t^{n}
$$

or

$$
\sum_{n \geq 1} h_{n, r} t^{n}-h_{2, r} t^{2}-h_{1, r} t=t\left(\sum_{n \geq 1} h_{n, r} t^{n}-h_{1, r} t\right)+t^{2} \sum_{n \geq 1} h_{n, r} t^{n} .
$$


Substituting $H_{r}(t)=\sum_{n=1}^{\infty} h_{n, r} t^{n}$ we get,

$$
H_{r}(t)-h_{2, r} t^{2}-h_{1, r} t=t\left(H_{r}(t)-h_{1, r} t\right)+t^{2} H_{r}(t) .
$$

Thus,

$$
H_{r}(t)=\frac{h_{1, r} t+t^{2}\left(h_{2, r}-h_{1, r}\right)}{1-t-t^{2}}
$$

\section{Properties of $r$-Sum Fibonacci Numbers}

In this section, we obtain further properties of $r$-sum Fibonacci numbers.

Proposition 4.1 (Sum of first $n$ terms). The sum of first $n$ terms of $r$-sum Fibonacci numbers is given by $h_{n+2, r}-h_{2, r}$.

Proof. By Binet's formula (3.1), we have

$$
\begin{aligned}
\sum_{k=1}^{n} h_{k, r} & =\frac{1}{\alpha-\beta}\left[\left(h_{2, r}-\beta h_{1, r}\right) \alpha^{0}-\left(h_{2, r}-\alpha h_{1, r}\right) \beta^{0}+\left(h_{2, r}-\beta h_{1, r}\right) \alpha^{1}-\left(h_{2, r}-\alpha h_{1, r}\right) \beta^{1}+\cdots+\left(h_{2, r}-\beta h_{1, r}\right) \alpha^{n-1}\right. \\
& \left.-\left(h_{2, r}-\alpha h_{1, r}\right) \beta^{n-1}\right] \\
& =\frac{1}{\alpha-\beta}\left[\left(h_{2, r}-\beta h_{1, r}\right)\left(1+\alpha+\cdots+\alpha^{n-1}\right)-\left(h_{2, r}-\alpha h_{1, r}\right)\left(1+\beta+\cdots+\beta^{n-1}\right)\right] \\
& =\frac{1}{\alpha-\beta}\left[\left(h_{2, r}-\beta h_{1, r}\right) \frac{\alpha^{n}-1}{\alpha-1}-\left(h_{2, r}-\alpha h_{1, r}\right) \frac{\beta^{n}-1}{\beta-1}\right] .
\end{aligned}
$$

Since $\alpha-1=-\beta$ and $\beta-1=-\alpha$, we have

$$
\sum_{k=1}^{n} h_{k, r}=\frac{1}{\alpha-\beta}\left[\frac{\left(h_{2, r}-\beta h_{1, r}\right)\left(\alpha^{n}-1\right) \alpha-\left(h_{2, r}-\alpha h_{1, r}\right)\left(\beta^{n}-1\right) \beta}{-\alpha \beta}\right] .
$$

Since $-\alpha \beta=1$, we get

$$
\begin{aligned}
\sum_{k=1}^{n} h_{k, r} & =\frac{1}{\alpha-\beta}\left[\left(h_{2, r}-\beta h_{1, r}\right)\left(\alpha^{n+1}-\alpha\right)-\left(h_{2, r}-\alpha h_{1, r}\right)\left(\beta^{n+1}-\beta\right)\right] \\
& =\frac{\left(h_{2, r}-\beta h_{1, r}\right) \alpha^{n+1}-\left(h_{2, r}-\alpha h_{1, r}\right) \beta^{n+1}}{\alpha-\beta}-\frac{\left(h_{2, r}-\beta h_{1, r}\right) \alpha-\left(h_{2, r}-\alpha h_{1, r}\right) \beta}{\alpha-\beta} \\
& =h_{n+2, r}-h_{2, r} .
\end{aligned}
$$

Proposition 4.2 (Sum of first $n$ terms with odd indices). The sum of the first $n$ terms with odd indices of $r$-sum Fibonacci numbers is given by $h_{2 n, r}-h_{2, r}+h_{1, r}$.

Proof. By Binet's formula (3.1), we have

$$
\begin{aligned}
\sum_{k=0}^{n-1} h_{2 k+1, r} & =\frac{1}{\alpha-\beta}\left[\left(h_{2, r}-\beta h_{1, r}\right) \alpha^{0}-\left(h_{2, r}-\alpha h_{1, r}\right) \beta^{0}+\left(h_{2, r}-\beta h_{1, r}\right) \alpha^{2}-\left(h_{2, r}-\alpha h_{1, r}\right) \beta^{2}\right. \\
& \left.+\cdots+\left(h_{2, r}-\beta h_{1, r}\right) \alpha^{2 n-2}-\left(h_{2, r}-\alpha h_{1, r}\right) \beta^{2 n-2}\right] \\
& =\frac{1}{\alpha-\beta}\left[\left(h_{2, r}-\beta h_{1, r}\right)\left(1+\alpha^{2}+\cdots+\alpha^{2 n-2}\right)-\left(h_{2, r}-\alpha h_{1, r}\right)\left(1+\beta^{2}+\cdots+\beta^{2 n-2}\right)\right] \\
& =\frac{1}{\alpha-\beta}\left[\frac{\left(h_{2, r}-\beta h_{1, r}\right)\left(\alpha^{2 n}-1\right)}{\alpha^{2}-1}-\frac{\left(h_{2, r}-\alpha h_{1, r}\right)\left(\beta^{2 n}-1\right)}{\beta^{2}-1}\right] .
\end{aligned}
$$


Since $\alpha^{2}-1=\alpha$ and $\beta^{2}-1=\beta$, we have

$$
\begin{aligned}
\sum_{k=0}^{n-1} h_{2 k+1} & =\frac{1}{\alpha-\beta}\left[\left(h_{2, r}-\beta h_{1, r}\right)\left(\alpha^{2 n-1}-\alpha^{-1}\right)-\left(h_{2, r}-\alpha h_{1, r}\right)\left(\beta^{2 n-1}-\beta^{-1}\right)\right] \\
& =\frac{\left(h_{2, r}-\beta h_{1, r}\right) \alpha^{2 n-1}-\left(h_{2, r}-\alpha h_{1, r}\right) \beta^{2 n-1}}{\alpha-\beta}-\frac{\left(h_{2, r}-\beta h_{1, r}\right) \alpha^{-1}-\left(h_{2, r}-\alpha h_{1, r}\right) \beta^{-1}}{\alpha-\beta} \\
& =h_{2 n, r}-h_{0, r} \\
& =h_{2 n, r}-h_{2, r}+h_{1, r} .
\end{aligned}
$$

Proposition 4.3 (Sum of first $n$ terms with even indices). The sum of the first $n$ terms with even indices of $r$-sum Fibonacci numbers is given by $h_{2 n+1, r}-h_{1, r}$.

Proof. By Binet's formula (3.1), we have

$$
\begin{aligned}
\sum_{k=1}^{n} h_{2 k, r} & =\frac{1}{\alpha-\beta}\left[\left(h_{2, r}-\beta h_{1, r}\right) \alpha^{1}-\left(h_{2, r}-\alpha h_{1, r}\right) \beta^{1}+\left(h_{2, r}-\beta h_{1, r}\right) \alpha^{3}-\left(h_{2, r}-\alpha h_{1, r}\right) \beta^{3}+\cdots+\left(h_{2, r}-\beta h_{1, r}\right) \alpha^{2 n-1}\right. \\
& \left.-\left(h_{2, r}-\alpha h_{1, r}\right) \beta^{2 n-1}\right] \\
& =\frac{1}{\alpha-\beta}\left[\left(h_{2, r}-\beta h_{1, r}\right)\left(\alpha+\alpha^{3}+\cdots+\alpha^{2 n-1}\right)-\left(h_{2, r}-\alpha h_{1, r}\right)\left(\beta+\beta^{3}+\cdots+\beta^{2 n-1}\right)\right] \\
& =\frac{1}{\alpha-\beta}\left[\left(h_{2, r}-\beta h_{1, r}\right) \frac{\alpha\left(\alpha^{2 n}-1\right)}{\alpha^{2}-1}-\left(h_{2, r}-\alpha h_{1, r}\right) \frac{\beta\left(\beta^{2 n}-1\right)}{\beta^{2}-1}\right] .
\end{aligned}
$$

Since $\alpha^{2}-1=\alpha$ and $\beta^{2}-1=\beta$, we get

$$
\begin{aligned}
\sum_{k=1}^{n} h_{2 k, r} & =\frac{1}{\alpha-\beta}\left[\left(h_{2, r}-\beta h_{1, r}\right)\left(\alpha^{2 n}-1\right)-\left(h_{2, r}-\alpha h_{1, r}\right)\left(\beta^{2 n}-1\right)\right] \\
& =\frac{\left(h_{2, r}-\beta h_{1, r}\right) \alpha^{2 n}-\left(h_{2, r}-\alpha h_{1, r}\right) \beta^{2 n}}{\alpha-\beta}-\frac{\left(h_{2, r}-\beta h_{1, r}\right)-\left(h_{2, r}-\alpha h_{1, r}\right)}{\alpha-\beta} \\
& =h_{2 n+1, r}-h_{1, r} .
\end{aligned}
$$

Proposition 4.4. For every positive integer n,

$$
h_{1, r}+h_{4, r}+h_{7, r}+\cdots+h_{3 n-2, r}=\frac{1}{2}\left(h_{3 n, r}-h_{2, r}+h_{1, r}\right) .
$$

Proof. By Binet's formula (3.1), we get

$$
\begin{aligned}
\sum_{k=1}^{n} h_{3 k-2, r} & =\frac{1}{\alpha-\beta}\left[\left(h_{2, r}-\beta h_{1, r}\right) \alpha^{0}-\left(h_{2, r}-\alpha h_{1, r}\right) \beta^{0}+\left(h_{2, r}-\beta h_{1, r}\right) \alpha^{3}-\left(h_{2, r}-\alpha h_{1, r}\right) \beta^{3}\right. \\
& \left.+\cdots+\left(h_{2, r}-\beta h_{1, r}\right) \alpha^{3 n-3}-\left(h_{2, r}-\alpha h_{1, r}\right) \beta^{3 n-3}\right] \\
& =\frac{1}{\alpha-\beta}\left[\left(h_{2, r}-\beta h_{1, r}\right)\left(1+\alpha^{3}+\cdots+\alpha^{3 n-3}\right)-\left(h_{2, r}-\alpha h_{1, r}\right)\left(1+\beta^{3}+\cdots+\beta^{3 n-3}\right)\right] \\
& =\frac{1}{\alpha-\beta}\left[\left(h_{2, r}-\beta h_{1, r}\right) \frac{\alpha^{3 n}-1}{\alpha^{3}-1}-\left(h_{2, r}-\alpha h_{0}\right) \frac{\beta^{3 n}-1}{\beta^{3}-1}\right] .
\end{aligned}
$$


Since $\alpha^{3}-1=2 \alpha$ and $\beta^{3}-1=2 \beta$, the above equation simplifies to

$$
\begin{aligned}
\sum_{k=1}^{n} h_{3 k-2, r} & =\frac{1}{2(\alpha-\beta)}\left[\left(h_{2, r}-\beta h_{1, r}\right)\left(\alpha^{3 n-1}-\alpha^{-1}\right)-\left(h_{2, r}-\alpha h_{1, r}\right)\left(\beta^{3 n-1}-\beta^{-1}\right)\right] \\
& =\frac{1}{2}\left[\frac{\left(h_{2, r}-\beta h_{1, r}\right) \alpha^{3 n-1}-\left(h_{2, r}-\alpha h_{1, r}\right) \beta^{3 n-1}}{\alpha-\beta}-\frac{\left(h_{2, r}-\beta h_{1, r}\right) \alpha^{-1}-\left(h_{2, r}-\alpha h_{1, r}\right) \beta^{-1}}{\alpha-\beta}\right] \\
& =\frac{1}{2}\left(h_{3 n, r}-h_{0, r}\right) \\
& =\frac{1}{2}\left(h_{3 n, r}-h_{2, r}+h_{1, r}\right) .
\end{aligned}
$$

Proposition 4.5. For every positive integer $n$,

$$
h_{2, r}+h_{5, r}+h_{8, r}+\cdots+h_{3 n-1, r}=\frac{1}{2}\left(h_{3 n+1, r}-h_{1, r}\right) .
$$

Proof. By Binet's formula (3.1), we have

$$
\begin{aligned}
\sum_{k=1}^{n} h_{3 k-1, r} & =\frac{1}{\alpha-\beta}\left[\left(h_{2, r}-\beta h_{1, r}\right) \alpha-\left(h_{2, r}-\alpha h_{1, r}\right) \beta+\left(h_{2, r}-\beta h_{1, r}\right) \alpha^{4}-\left(h_{2, r}-\alpha h_{1, r}\right) \beta^{4}\right. \\
& \left.+\cdots+\left(h_{2, r}-\beta h_{1, r}\right) \alpha^{3 n-2}-\left(h_{2, r}-\alpha h_{1, r}\right) \beta^{3 n-2}\right] \\
& =\frac{1}{\alpha-\beta}\left[\left(h_{2, r}-\beta h_{1, r}\right)\left(\alpha+\alpha^{4}+\cdots+\alpha^{3 n-2}\right)-\left(h_{2, r}-\alpha h_{1, r}\right)\left(\beta+\beta^{4}+\cdots+\beta^{3 n-2}\right)\right] \\
& =\frac{1}{\alpha-\beta}\left[\left(h_{2, r}-\beta h_{1, r}\right) \frac{\alpha^{3 n+1}-\alpha}{\alpha^{3}-1}-\left(h_{2, r}-\alpha h_{1, r}\right) \frac{\beta^{3 n+1}-\beta}{\beta^{3}-1}\right] .
\end{aligned}
$$

Since $\alpha^{3}-1=2 \alpha$ and $\beta^{3}-1=2 \beta$, then

$$
\begin{aligned}
\sum_{k=1}^{n} h_{3 k-1, r} & =\frac{1}{2(\alpha-\beta)}\left[\left(h_{2, r}-\beta h_{1, r}\right)\left(\alpha^{3 n}-1\right)-\left(h_{2, r}-\alpha h_{1, r}\right)\left(\beta^{3 n}-1\right)\right] \\
& =\frac{1}{2}\left[\frac{\left(h_{2, r}-\beta h_{1, r}\right) \alpha^{3 n}-\left(h_{2, r}-\alpha h_{1, r}\right) \beta^{3 n}}{\alpha-\beta}-\frac{\left(h_{2, r}-\beta h_{1, r}\right)-\left(h_{2, r}-\alpha h_{1, r}\right)}{\alpha-\beta}\right] \\
& =\frac{1}{2}\left(h_{3 n+1, r}-h_{1, r}\right) .
\end{aligned}
$$

Proposition 4.6. For every positive integer $n$,

$$
h_{3, r}+h_{6, r}+h_{9, r}+\cdots+h_{3 n, r}=\frac{1}{2}\left(h_{3 n+2, r}-h_{2, r}\right) .
$$

Proof. By Binet's formula (3.1), we obtain

$$
\begin{aligned}
\sum_{k=1}^{n} h_{3 k, r} & =\frac{1}{\alpha-\beta}\left[\left(h_{1, n}-\beta h_{1, r}\right) \alpha^{2}-\left(h_{2, r}-\alpha h_{1, r}\right) \beta^{2}+\left(h_{2, r}-\beta h_{1, r}\right) \alpha^{5}-\left(h_{2, r}-\alpha h_{1, r}\right) \beta^{5}+\cdots+\left(h_{2, r}-\beta h_{1, r}\right) \alpha^{3 n-1}\right. \\
& \left.-\left(h_{2, r}-\alpha h_{1, r}\right) \beta^{3 n-1}\right] \\
& =\frac{1}{\alpha-\beta}\left[\left(h_{2, r}-\beta h_{1, r}\right)\left(\alpha^{2}+\alpha^{5}+\cdots+\alpha^{3 n-1}\right)-\left(h_{2, r}-\alpha h_{1, r}\right)\left(\beta^{2}+\beta^{5}+\cdots+\beta^{3 n-1}\right)\right] \\
& =\frac{1}{\alpha-\beta}\left[\left(h_{2, r}-\beta h_{1, r}\right) \frac{\alpha^{3 n+2}-\alpha^{2}}{\alpha^{3}-1}-\left(h_{2, r}-\alpha h_{1, r}\right) \frac{\beta^{3 n+2}-\beta^{2}}{\beta^{3}-1}\right]
\end{aligned}
$$


Since $\alpha^{3}-1=2 \alpha$ and $\beta^{3}-1=2 \beta$, we get

$$
\begin{aligned}
\sum_{k=1}^{n} h_{3 k, r} & =\frac{1}{2(\alpha-\beta)}\left[\left(h_{2, r}-\beta h_{1, r}\right)\left(\alpha^{3 n+1}-\alpha\right)-\left(h_{2, r}-\alpha h_{1, r}\right)\left(\beta^{3 n+1}-\beta\right)\right] \\
& =\frac{1}{2}\left[\frac{\left(h_{2, r}-\beta h_{1, r}\right) \alpha^{3 n+1}-\left(h_{2, r}-\alpha h_{1, r}\right) \beta^{3 n+1}}{\alpha-\beta}-\frac{\left(h_{2, r}-\beta h_{1, r}\right) \alpha-\left(h_{2, r}-\alpha h_{1, r}\right) \beta}{\alpha-\beta}\right] \\
& =\frac{1}{2}\left(h_{3 n+2, r}-h_{2, r}\right) .
\end{aligned}
$$

Proposition 4.7 (Alternating sum formula). For every positive integer $n$,

$$
\sum_{k=1}^{n}(-1)^{k+1} h_{k, r}=(-1)^{n-1} h_{n-1, r}+2 h_{1, r}-h_{2, r} .
$$

Proof. By Binet's formula (3.1), we get

$$
\begin{aligned}
\sum_{k=1}^{n}(-1)^{k+1} h_{k, r} & =\frac{1}{\alpha-\beta}\left[\left(h_{2, r}-\beta h_{1, r}\right)-\left(h_{2, r}-\alpha h_{1, r}\right)-\left(\left(h_{2, r}-\beta h_{1, r}\right) \alpha-\left(h_{2, r}-\alpha h_{1, r}\right) \beta\right)\right. \\
& \left.+\cdots+(-1)^{n+1}\left(\left(h_{2, r}-\beta h_{1, r}\right) \alpha^{n-1}-\left(h_{2, r}-\alpha h_{1, r}\right) \beta^{n-1}\right)\right] \\
& =\frac{1}{\alpha-\beta}\left[\left(h_{2, r}-\beta h_{1, r}\right)\left(1-\alpha+\cdots+(-1)^{n+1} \alpha^{n-1}\right)-\left(\left(h_{2, r}-\alpha h_{1, r}\right)\left(1-\beta+\cdots+(-1)^{n+1} \beta^{n-1}\right)\right]\right. \\
& =\frac{1}{\alpha-\beta}\left[\left(h_{2, r}-\beta h_{1, r}\right) \frac{\left((-\alpha)^{n}-1\right)}{-\alpha-1}-\left(h_{2, r}-\alpha h_{1, r}\right) \frac{\left((-\beta)^{n}-1\right)}{-\beta-1}\right] .
\end{aligned}
$$

Since $-\alpha-1=-\alpha^{2}$ and $-\beta-1=-\beta^{2}$, we have

$$
\begin{aligned}
\sum_{k=1}^{n}(-1)^{k+1} h_{k, r} & =\frac{1}{\alpha-\beta}\left[\left(h_{2, r}-\beta h_{1, r}\right) \frac{(-1)^{n} \alpha^{n}-1}{-\alpha^{2}}-\left(h_{2, r}-\alpha h_{1, r}\right) \frac{(-1)^{n} \beta^{n}-1}{-\beta^{2}}\right] \\
& =(-1)^{n-1} \frac{1}{\alpha-\beta}\left[\left(h_{2, r}-\beta h_{1, r}\right) \alpha^{n-2}-\left(h_{2, r}-\alpha h_{1, r}\right) \beta^{n-2}\right] \\
& +\frac{1}{\alpha-\beta}\left[\left(h_{2, r}-\beta h_{1, r}\right) \alpha^{-2}-\left(h_{2, r}-\alpha h_{1, r}\right) \beta^{-2}\right] \\
& =(-1)^{n-1} h_{n-1, r}+\frac{1}{\alpha-\beta}\left[\frac{\left(h_{2, r}-\beta h_{1, r}\right)}{\alpha^{2}}-\frac{\left(h_{2, r}-\alpha h_{1, r}\right)}{\beta^{2}}\right] .
\end{aligned}
$$

This gives,

$$
\begin{aligned}
\sum_{k=1}^{n}(-1)^{k+1} h_{k, r} & =(-1)^{n-1} h_{n-1, r}+\frac{1}{(\alpha \beta)^{2}(\alpha-\beta)}\left[\left(h_{2, r}-\beta h_{1, r}\right) \beta^{2}-\left(h_{2, r}-\alpha h_{1, r}\right) \alpha^{2}\right] \\
& =(-1)^{n-1} h_{n-1, r}+\left[h_{1, r}\left(\frac{\alpha^{3}-\beta^{3}}{\alpha-\beta}\right)-h_{2, r}\left(\frac{\alpha^{2}-\beta^{2}}{\alpha-\beta}\right)\right] \\
& =(-1)^{n-1} h_{n-1, r}+\left[h_{1, r}\left(\frac{2(\alpha-\beta)}{\alpha-\beta}\right)-h_{2, r}\left(\frac{\alpha^{2}-\beta^{2}}{\alpha-\beta}\right)\right] .
\end{aligned}
$$

Since $\alpha-\beta=\sqrt{5}$ and $\alpha^{2}-\beta^{2}=\sqrt{5}$, then

$$
\sum_{k=1}^{n}(-1)^{k+1} h_{k, r}=(-1)^{n-1} h_{n-1, r}+2 h_{1, r}-h_{2, r} .
$$

Proposition 4.8. For every positive integer $n$,

$$
h_{2 n, r}=\sum_{k=0}^{n}\left(\begin{array}{l}
n \\
k
\end{array}\right) h_{k, r} \text {. }
$$


Proof. By Binet's formula (3.1), we get

$$
\begin{aligned}
h_{2 n, r} & =\frac{1}{\alpha-\beta}\left[\left(h_{2, r}-\beta h_{1, r}\right) \alpha^{2 n-1}-\left(h_{2, r}-\alpha h_{1, r}\right) \beta^{2 n-1}\right] \\
& =\frac{1}{\alpha-\beta}\left[\left(h_{2, r}-\beta h_{1, r}\right) \frac{\alpha^{2 n}}{\alpha}-\left(h_{2, r}-\alpha h_{1, r}\right) \frac{\beta^{2 n}}{\beta}\right] .
\end{aligned}
$$

Since $\alpha^{2}=1+\alpha$ and $\beta^{2}=1+\beta$, then

$$
h_{2 n, r}=\frac{1}{\alpha-\beta}\left[\left(h_{2, r}-\beta h_{1, r}\right) \frac{(1+\alpha)^{n}}{\alpha}-\left(h_{2, r}-\alpha h_{1, r}\right) \frac{(1+\beta)^{n}}{\beta}\right] .
$$

Since $(1+x)^{n}=\sum_{k=0}^{n}\left(\begin{array}{l}n \\ k\end{array}\right) x^{k}$, we have

$$
\begin{aligned}
h_{2 n, r} & =\frac{1}{\alpha-\beta}\left[\left(h_{2, r}-\beta h_{1, r}\right) \sum_{k=0}^{n}\left(\begin{array}{l}
n \\
k
\end{array}\right) \alpha^{k-1}-\left(h_{2, r}-\alpha h_{1, r}\right) \sum_{k=0}^{n}\left(\begin{array}{l}
n \\
k
\end{array}\right) \beta^{k-1}\right] \\
& =\sum_{k=0}^{n}\left(\begin{array}{l}
n \\
k
\end{array}\right)\left[\frac{\left(h_{2, r}-\beta h_{o, r}\right) \alpha^{k-1}-\left(h_{2, r}-\alpha h_{1, r}\right) \beta^{k-1}}{\alpha-\beta}\right] \\
& =\sum_{k=0}^{n}\left(\begin{array}{l}
n \\
k
\end{array}\right) h_{k, r} .
\end{aligned}
$$

Proposition 4.9 (Explicit sum formula). For every positive integer n,

$$
h_{n, r}=h_{1, r} \sum_{k=0}^{\left\lfloor\frac{n-1}{2}\right\rfloor}\left(\begin{array}{c}
n-k-1 \\
k
\end{array}\right)+\left(h_{2, r}-h_{1, r}\right) \sum_{k=0}^{\left\lfloor\frac{n-2}{2}\right\rfloor}\left(\begin{array}{c}
n-k-2 \\
k
\end{array}\right),
$$

where $\lfloor n\rfloor$ is the greatest integer less than or equal to $n$.

Proof. By generating function (3.3), we have

$$
\begin{aligned}
\sum_{n=1}^{\infty} h_{n, r} t^{n} & =\frac{h_{1, r} t+t^{2}\left(h_{2, r}-h_{1, r}\right)}{1-t-t^{2}} \\
& =t\left[h_{1, r}+t\left(h_{2, r}-h_{1, r}\right)\right]\left(1-t-t^{2}\right)^{-1} \\
& =t\left[h_{1, r}+t\left(h_{2, r}-h_{1, r}\right)\right]\left[1-\left(t+t^{2}\right)\right]^{-1} \\
& =t\left[h_{1, r}+t\left(h_{2, r}-h_{1, r}\right)\right] \sum_{n=1}^{\infty} t^{n-1}(1+t)^{n-1} \\
& =\left[h_{1, r}+t\left(h_{2, r}-h_{1, r}\right)\right] \sum_{n=1}^{\infty} t^{n} \sum_{k=0}^{n-1}\left(\begin{array}{c}
n-1 \\
k
\end{array}\right) t^{k} \\
& =\left[h_{1, r}+t\left(h_{2, r}-h_{1, r}\right)\right] \sum_{n=1}^{\infty} \sum_{k=0}^{n-1} \frac{(n-1) !}{k !(n-k-1) !} t^{n+k} .
\end{aligned}
$$

Replacing $n$ by $n+k+1$, we get

$$
\sum_{n=1}^{\infty} h_{n, r} t^{n}=\left[h_{1, r}+t\left(h_{2, r}-h_{1, r}\right)\right] \sum_{n=1}^{\infty} \sum_{k=0}^{\infty} \frac{(n+k) !}{k ! n !} t^{n+2 k+1} .
$$

Now, replacing $n$ by $n-2 k-1$, we have

$$
\begin{aligned}
\sum_{n=1}^{\infty} h_{n, r} t^{n} & =\left[h_{1, r}+t\left(h_{2, r}-h_{1, r}\right)\right] \sum_{n=1}^{\infty} \sum_{k=0}^{\left\lfloor\frac{n-1}{2}\right\rfloor} \frac{(n-k-1) !}{k !(n-2 k-1) !} t^{n} \\
& =h_{1, r}\left[\sum_{n=1}^{\infty} \sum_{k=0}^{\left\lfloor\frac{n-1}{2}\right\rfloor} \frac{(n-k-1) !}{k !(n-2 k-1) !}\right] t^{n}+\left(h_{2, r}-h_{1, r}\right) \sum_{n=1}^{\infty}\left[\sum_{k=0}^{\left\lfloor\frac{n-1}{2}\right\rfloor} \frac{(n-k-1) !}{k !(n-2 k-1) !}\right] t^{n+1} .
\end{aligned}
$$


Equating the coefficients of $t^{n}$, we obtain

$$
h_{n, r}=h_{1, r} \sum_{k=0}^{\left\lfloor\frac{n-1}{2}\right\rfloor}\left(\begin{array}{c}
n-k-1 \\
k
\end{array}\right)+\left(h_{2, r}-h_{1, r}\right) \sum_{k=0}^{\left\lfloor\frac{n-2}{2}\right\rfloor}\left(\begin{array}{c}
n-k-2 \\
k
\end{array}\right) .
$$

Hence the proof follows.

Proposition 4.10. For every positive integer $n$,

$$
h_{-n, r}=(-1)^{n}\left(h_{2, r} f_{n+2}-h_{1, r} f_{n+3}\right) .
$$

Proof. By Binet's formula (3.1), we have

$$
\begin{aligned}
h_{-n, r} & =\frac{1}{\alpha-\beta}\left[\left(h_{2, r}-\beta h_{1, r}\right) \alpha^{-n-1}-\left(h_{2, r}-\alpha h_{1, r}\right) \beta^{-n-1}\right] \\
& =\frac{1}{\alpha-\beta}\left[\left(h_{2, r}-\beta h_{1, r}\right) \frac{1}{\alpha^{n+1}}-\left(h_{2, r}-\alpha h_{1, r}\right) \frac{1}{\beta^{n+1}}\right] .
\end{aligned}
$$

Since $\frac{1}{\alpha}=-\beta$ and $\frac{1}{\beta}=-\alpha$, we have

$$
\begin{aligned}
h_{-n, r} & =\frac{1}{\alpha-\beta}\left[\left(h_{2, r}-\beta h_{1, r}\right)(-1)^{n+1} \beta^{n+1}-\left(h_{2, r}-\alpha h_{1, r}\right)(-1)^{n+1} \alpha^{n+1}\right] \\
& =\frac{(-1)^{n+1}}{\alpha-\beta}\left[\left(h_{2, r}-\beta h_{1, r}\right) \beta^{n+1}-\left(h_{2, r}-\alpha h_{1, r}\right) \alpha^{n+1}\right] \\
& =\frac{(-1)^{n+1}}{\alpha-\beta}\left[h_{2, r} \beta^{n+1}-h_{1, r} \beta^{n+2}-h_{2, r} \alpha^{n+1}+h_{1, r} \alpha^{n+2}\right] \\
& =\frac{(-1)^{n+2}}{\alpha-\beta}\left[h_{2, r}\left(\alpha^{n+1}-\beta^{n+1}\right)-h_{1, r}\left(\alpha^{n+2}-\beta^{n+2}\right)\right] \\
& =(-1)^{n+2}\left[\frac{h_{2, r}\left(\alpha^{n+1}-\beta^{n+1}\right)}{\alpha-\beta}-\frac{h_{1, r}\left(\alpha^{n+2}-\beta^{n+2}\right)}{\alpha-\beta}\right] \\
& =(-1)^{n+2}\left(h_{2, r} f_{n+2}-h_{1, r} f_{n+3}\right) \\
& =(-1)^{n}\left(h_{2, r} f_{n+2}-h_{1, r} f_{n+3}\right) .
\end{aligned}
$$

Proposition 4.11 (Honsberger's identity). If $n>m$ then

$$
h_{n+m, r}=h_{n, r} f_{m}+h_{n+1, r} f_{m+1},
$$

for all $m \geq 0$ and $n>0$.

Proof. Since by Corollary 3.2, we have

$$
h_{n+m, r}=h_{2, r} f_{n+m}+h_{1, r} f_{n+m-1},
$$

then by Honsberger's identity of Fibonacci numbers (1.4), we get

$$
\begin{aligned}
h_{n+m, r} & =h_{2, r}\left(f_{n} f_{m}+f_{n+1} f_{m+1}\right)+h_{1, r}\left(f_{n-1} f_{m}+f_{n} f_{m+1}\right) \\
& =f_{m}\left(h_{2, r} f_{n}+h_{1, r} f_{n-1}\right)+f_{m+1}\left(h_{2, r} f_{n+1}+h_{1, r} f_{n}\right) .
\end{aligned}
$$

Applying Corollary 3.2 again we have, $h_{n+m, r}=h_{n, r} f_{m}+h_{n+1, r} f_{m+1}$.

Corollary 4.12. We have:

1. $h_{2 n, r}=h_{n, r} f_{n}+h_{n+1, r} f_{n+1}$, 
2. $h_{2 n-1, r}=h_{n, r} f_{n-1}+h_{n+1, r} f_{n}$,

3. $h_{2 n-2, r}=h_{n, r} f_{n-2}+h_{n+1} f_{n-1}$,

4. $h_{2 n-k, r}=h_{n, r} f_{n-k}+h_{n+1, r}+f_{n-k+1}$.

Proof. The results follow from Proposition 4.11 upon setting $m=n, m=n-1, m=n-2$, and $m=n-k$ in that order.

Proposition 4.13. For every $n \geq 2$, we have

$$
h_{2, r} h_{3, r}+h_{3, r} h_{4, r}+\cdots+h_{2 n-1, r} h_{2 n, r}=h_{2 n, r}^{2}-h_{2, r}^{2} .
$$

Proof. We induct on $n$. For base case, $n=2$ :

The left hand side gives

$$
h_{2, r} h_{3, r}+h_{3, r} h_{4, r}=h_{3, r}\left(h_{2, r}+h_{4, r}\right)
$$

while the right hand side gives

$$
h_{4, r}^{2}-h_{2, r}^{2}=\left(h_{4, r}-h_{2, r}\right)\left(h_{4, r}+h_{2, r}\right)=h_{3, r}\left(h_{4, r}+h_{2, r}\right) .
$$

Since the left hand side equals to the right hand side, the base case holds.

For the induction step, we will assume the formula holds true for $n$ and prove that it holds true for $n+1$.

Since by inductive hypothesis

$$
h_{2, r} h_{3, r}+h_{3, r} h_{4, r}+\cdots+h_{2 n-1, r} h_{2 n, r}=h_{2 n, r}^{2}-h_{2, r}^{2},
$$

then

$$
\begin{aligned}
h_{2, r} h_{3, r}+h_{3, r} h_{4, r}+\cdots+h_{2 n-1, r} h_{2 n, r}+h_{2 n} h_{2 n+1, r}+h_{2 n+1, r} h_{2 n+2, r} & =h_{2 n, r}^{2}-h_{2, r}^{2}+h_{2 n, r} h_{2 n+1, r}+h_{2 n+1, r} h_{2 n+2, r} \\
& =h_{2 n, r}^{2}+h_{2 n, r} h_{2 n+1, r}-h_{2, r}^{2}+h_{2 n+1, r} h_{2 n+2, r} \\
& =h_{2 n, r}\left(h_{2 n, r}+h_{2 n+1, r}\right)+h_{2 n+1, r} h_{2 n+2, r}-h_{2, r}^{2} \\
& =h_{2 n, r} h_{2 n+2, r}+h_{2 n+1, r} h_{2 n+2, r}-h_{2, r}^{2} \\
& =h_{2 n+2, r}\left(h_{2 n, r}+h_{2 n+1, r}\right)-h_{2, r}^{2} \\
& =h_{2 n+2, r}^{2}-h_{2, r}^{2} .
\end{aligned}
$$

By the principle of mathematical induction, the result follows.

Lemma 4.14. The $n^{\text {th }}$ Fibonacci number, $f_{n}$, is given by

$$
f_{n}=\frac{h_{2, r} h_{n, r}-h_{1, r} h_{n+1, r}}{h_{2, r}^{2}-h_{1, r} h_{3, r}} .
$$

Proof. We have, by Binet's formula (3.1), that

$$
\begin{aligned}
h_{2, r} h_{n, r}-h_{1, r} h_{n+1, r} & =h_{2, r}\left[\frac{\left(h_{2, r}-\beta h_{1, r}\right) \alpha^{n-1}}{\alpha-\beta}+\frac{\left(\alpha h_{1, r}-h_{2, r}\right) \beta^{n-1}}{\alpha-\beta}\right]-h_{1, r}\left[\frac{\left(h_{2, r}-\beta h_{1, r}\right) \alpha^{n}}{\alpha-\beta}+\frac{\left(\alpha h_{1, r}-h_{2, r}\right) \beta^{n}}{\alpha-\beta}\right] \\
& =h_{2, r}\left[h_{2, r} \frac{\left(\alpha^{n-1}-\beta^{n-1}\right)}{\alpha-\beta}+h_{1, r} \frac{\left(\alpha^{n-2}-\beta^{n-2}\right)}{\alpha-\beta}\right]-h_{1, r}\left[h_{2, r} \frac{\left(\alpha^{n}-\beta^{n}\right)}{\alpha-\beta}+h_{1, r} \frac{\left(\alpha^{n-1}-\beta^{n-1}\right)}{\alpha-\beta}\right] \\
& =\frac{\alpha^{n-1}-\beta^{n-1}}{\alpha-\beta}\left[h_{2, r}^{2}-h_{1, r}^{2}\right]+\frac{h_{1, r} h_{2, r}}{\alpha-\beta}\left[\alpha^{n-2}-\beta^{n-2}-\alpha^{n}+\beta^{n}\right] \\
& =\frac{\alpha^{n-1}-\beta^{n-1}}{\alpha-\beta}\left[h_{2, r}^{2}-h_{1, r}^{2}-h_{1, r} h_{2, r}\right] .
\end{aligned}
$$

Now,

$$
f_{n}=\frac{\alpha^{n-1}-\beta^{n-1}}{\alpha-\beta}=\frac{h_{2, r} h_{n, r}-h_{1, r} h_{n+1, r}}{h_{2, r}^{2}-h_{1, r} h_{3, r}} .
$$


Theorem 4.15 (Generalized identity). Let $h_{n, r}$ be the $n^{\text {th }}$ term of $r$-sum Fibonacci sequence then

$$
h_{m, r} h_{n, r}-h_{m-k, r} h_{n+k, r}=\frac{(-1)^{m-k-1}}{h_{2, r}^{2}-h_{1, r} h_{3, r}}\left[\left(h_{2, r} h_{k+1, r}-h_{1, r} h_{k+2, r}\right)\left(h_{2, r} h_{n-m+k+1, r}-h_{1, r} h_{n-m+k+2, r}\right)\right],
$$

where $n \geq m$ and $k \geq 1$.

Proof. By Binet's formula (3.1), we have

$$
h_{n, r}=A \alpha^{n-1}+B \beta^{n-1}
$$

where $A=\frac{h_{2, r}-\beta h_{1, r}}{\alpha-\beta}, B=\frac{\alpha h_{1, r}-h_{2, r}}{\alpha-\beta}, \alpha=\frac{1+\sqrt{5}}{2}$ and $\beta=\frac{1-\sqrt{5}}{2}$.

Now,

$$
\begin{aligned}
h_{m, r} h_{n, r}-h_{m-k, r} h_{n+k, r} & =\left(A \alpha^{m-1}+B \beta^{m-1}\right)\left(A \alpha^{n-1}+B \beta^{n-1}\right)-\left(A \alpha^{m-k-1}+B \beta^{m-k-1}\right)\left(A \alpha^{n+k-1}+B \beta^{n+k-1}\right) \\
& =A B\left(\alpha^{k}-\beta^{k}\right)\left[\frac{\alpha^{m-1} \beta^{n-1}}{\alpha^{k}}-\frac{\alpha^{n-1} \beta^{m-1}}{\beta^{k}}\right] \\
& =A B(-1)^{-k}\left(\alpha^{k}-\beta^{k}\right)\left(\alpha^{m-1} \beta^{m-1}\right)\left(\beta^{n-m+k}-\alpha^{n-m+k}\right) \\
& =-A B(-1)^{m-k-1}\left(\alpha^{k}-\beta^{k}\right)\left(\alpha^{n-m+k}-\beta^{n-m+k}\right) .
\end{aligned}
$$

Since $-A B=\frac{h_{2, r}^{2}-h_{1, r} h_{3, r}}{(\alpha-\beta)^{2}}$, then

$$
\begin{aligned}
h_{m, r} h_{n, r}-h_{m-k, r} h_{n+k, r} & =\frac{h_{2, r}^{2}-h_{1, r} h_{3, r}}{(\alpha-\beta)^{2}}(-1)^{m-k-1}\left[\left(\alpha^{k}-\beta^{k}\right)\left(\alpha^{n-m+k}-\beta^{n-m+k}\right)\right] \\
& =\left(h_{2, r}^{2}-h_{1, r} h_{3, r}\right)(-1)^{m-k-1}\left[\frac{\alpha^{k}-\beta^{k}}{\alpha-\beta}\left(\frac{\alpha^{n-m+k}-\beta^{n-m+k}}{\alpha-\beta}\right)\right] .
\end{aligned}
$$

By Lemma 4.14, we have

$$
f_{k+1}=\frac{\alpha^{k}-\beta^{k}}{\alpha-\beta}=\frac{h_{2, r} h_{k+1, r}-h_{1, r} h_{k+2, r}}{h_{2, r}^{2}-h_{1, r} h_{3, r}}
$$

and

$$
f_{n-m+k+1}=\frac{\alpha^{n-m+k}-\beta^{n-m+k}}{\alpha-\beta}=\frac{h_{2, r} h_{n-m+k+1, r}-h_{1, r} h_{n-m+k+2, r}}{h_{2, r}^{2}-h_{1, r} h_{3, r}} .
$$

So

$$
h_{m, r} h_{n, r}-h_{m-k, r} h_{n+k, r}=(-1)^{m-k-1}\left[\frac{\left(h_{2, r} h_{k+1, r}-h_{1, r} h_{k+2, r}\right)\left(h_{2, r} h_{n-m+k+1, r}-h_{1, r} h_{n-m+k+2}\right)}{h_{2, r}^{2}-h_{1, r} h_{3, r}}\right] .
$$

Hence the proof follows.

Corollary 4.16 (Catalan's identity). If we take $m=n$ in the generalized identity (4.2), we obtain

$$
h_{n, r}^{2}-h_{n-k, r} h_{n+k, r}=\frac{(-1)^{n-k-1}}{h_{2, r}^{2}-h_{1, r} h_{3, r}}\left[h_{2, r} h_{k+1, r}-h_{1, r} h_{k+2, r}\right]^{2},
$$

for all $n>k \geq 1$.

Corollary 4.17 (Cassini's identity). If $m=n$ and $k=1$ in the generalized identity (4.2), then

$$
h_{n, r}^{2}-h_{n-1, r} h_{n+1, r}=(-1)^{n-2}\left(h_{2, r}^{2}-h_{1, r} h_{3, r}\right),
$$

for all $n \geq 1$.

Corollary 4.18 (d'Ocagne's identity). If $n=m, m=n+1$ and $k=1$ in the generalized identity (4.2), then

$$
h_{n+1, r} h_{m, r}-h_{n, r} h_{m+1, r}=(-1)^{n-1}\left[h_{2, r} h_{m-n+1, r}-h_{1, r} h_{m-n+2, r}\right],
$$

where $m>n \geq 0$. 


\section{Determinant Identities}

Determinants play a significant role in various areas in mathematics. For instance, they are quite useful in analysis and solution of systems of linear equations. T. Koshy [6] devoted two chapters of his book to the use of matrices and determinants in Fibonacci numbers. In this section, we obtain further properties of $r$-sum Fibonacci numbers involving determinants.

Proposition 5.1. For every positive integer $n$,

$$
\left|\begin{array}{lll}
h_{n+1, r} & h_{n+2, r} & h_{n+3, r} \\
h_{n+4, r} & h_{n+5, r} & h_{n+6, r} \\
h_{n+7, r} & h_{n+8, r} & h_{n+9, r}
\end{array}\right|=0 .
$$

Proof. Applying column reduction $C_{1} \longrightarrow C_{1}+C_{2}$ to the matrix, i.e., replace the entries of column 1 with the sum of the entries of columns 1 and 2, we get that two columns are identical and hence the determinant of the matrix is zero.

Proposition 5.2. For every positive integer $n$,

$$
\left|\begin{array}{ccc}
h_{n, r}+h_{n+1, r} & h_{n+1, r}+h_{n+2, r} & h_{n+2, r}+h_{n, r} \\
h_{n+2, r} & h_{n, r} & h_{n+1, r} \\
1 & 1 & 1
\end{array}\right|=0 .
$$

Proof. Applying $R_{1} \longrightarrow R_{1}+R_{2}$, we get that the determinant of the matrix is

$$
\left|\begin{array}{ccc}
2 h_{n+2, r} & 2 h_{n+2, r} & 2 h_{n+2, r} \\
h_{n+2, r} & h_{n, r} & h_{n+1, r} \\
1 & 1 & 1
\end{array}\right|=2 h_{n+2, r}\left|\begin{array}{ccc}
1 & 1 & 1 \\
h_{n+2, r} & h_{n, r} & h_{n+1, r} \\
1 & 1 & 1
\end{array}\right| .
$$

Since two rows are identical, the determinant is zero.

Proposition 5.3. Let $n$ be a positive integer, then

$$
\left|\begin{array}{ccc}
h_{n, r} & f_{n} & 1 \\
h_{n+1, r} & f_{n+1} & 1 \\
h_{n+2, r} & f_{n+2} & 1
\end{array}\right|=f_{n} h_{n+1, r}-f_{n+1} h_{n, r} .
$$

Proof. Applying $R_{1} \longrightarrow R_{2}-R_{1}$ and $R_{2} \longrightarrow R_{3}-R_{2}$, we get that

$$
\left|\begin{array}{ccc}
h_{n, r} & f_{n} & 1 \\
h_{n+1, r} & f_{n+1} & 1 \\
h_{n+2, r} & f_{n+2} & 1
\end{array}\right|=\left|\begin{array}{ccc}
h_{n+1, r}-h_{n, r} & f_{n+1}-f_{n} & 0 \\
h_{n, r} & f_{n} & 0 \\
h_{n+2, r} & f_{n+2} & 1
\end{array}\right| .
$$

The result is thus immediate.

Proposition 5.4. For every positive integer $n$,

$$
\left|\begin{array}{ccc}
h_{n, r} & l_{n} & 1 \\
h_{n+1, r} & l_{n+1} & 1 \\
h_{n+2, r} & l_{n+2} & 1
\end{array}\right|=l_{n} h_{n+1, r}-l_{n+1} h_{n, r}
$$

Proof. The proof follows as in the proof of Proposition 5.3.

Proposition 5.5. For every positive integer $n$,

$$
\left|\begin{array}{cccc}
1+h_{n, r} & h_{n+1, r} & \cdots & h_{n+p, r} \\
h_{n, r} & 1+h_{n+1, r} & \cdots & h_{n+p, r} \\
\vdots & \vdots & \ddots & \vdots \\
h_{n, r} & h_{n+1, r} & \cdots & 1+h_{n+p, r}
\end{array}\right|=1+h_{n, r}+h_{n+1, r}+\cdots+h_{n+p, r}
$$

Proof. The proof follows by induction on $n$ and making use of column reductions. 
Proposition 5.6. Let $n$ be a positive integer, then

$$
\left|\begin{array}{ccc}
h_{n, r} & h_{n+1, r} & h_{n+2, r} \\
h_{n+2, r} & h_{n, r} & h_{n+1, r} \\
h_{n+1, r} & h_{n+2, r} & h_{n, r}
\end{array}\right|=2\left(h_{n, r}^{3}+h_{n+1, r}^{3}\right) .
$$

Proof. We have

$$
\begin{aligned}
\left|\begin{array}{ccc}
h_{n, r} & h_{n+1, r} & h_{n+2, r} \\
h_{n+2, r} & h_{n, r} & h_{n+1, r} \\
h_{n+1, r} & h_{n+2, r} & h_{n, r}
\end{array}\right| & =h_{n, r}\left(h_{n, r}^{2}-h_{n+1, r} h_{n+2, r}\right)+h_{n+1, r}\left(h_{n+1, r}^{2}-h_{n, r} h_{n+2, r}\right)+h_{n+2, r}\left(h_{n+2, r}^{2}-h_{n, r} h_{n+1, r}\right) \\
& =h_{n, r}^{3}+h_{n+1, r}^{3}+h_{n+2, r}^{3}-3 h_{n, r} h_{n+1, r} h_{n+2, r} .
\end{aligned}
$$

Substituting $h_{n+2, r}=h_{n, r}+h_{n+1, r}$ and expanding, we obtain the desired result.

\section{Conclusion}

In this paper, we have derived Binet's formula (3.1) and generating function (3.3) for the $r$-sum Fibonacci sequence. Further, we have obtained explicit sum formula, sum of first $n$ terms, sum of first $n$ terms with even indices, sum of first $n$ terms with odd indices, alternating sum of $n$ terms of $r$-sum Fibonacci sequence, Honsberger's identity, determinant identities and generalized identity (4.2) from which Cassini's identity, Catalan's identity and d'Ocagne's identity are simple cases.

\section{References}

[1] S. Falcon, A. Plaza, On the Fibonacci K-numbers, Chaos Solution Fractals, 32(5) (2007), 1615-1624.

[2] Y.K Gupta, M. Singh, O. Sikhwal, Generalized Fibonacci-Like sequence associated with Fibonacci and Lucas sequences, Turkish J. Anal. Number Theory, 2(6) (2014), 233-238.

[3] A.F. Horadam, A generalized Fibonacci sequence, Amer. Math. Monthly, 68(1961), 455-459.

[4] A.F. Horadam, Basic properties of a certain generalized sequence of numbers, Fib. Quart, 3(3) (1965),161-176.

[5] D. Kalma, R. Mena, The Fibonacci Numbers-Exposed, Math. Mag., 2 (2002).

[6] T. Koshy, Fibonacci and Lucas Numbers with Applications, Wisley-Interscience Publications, New York, 2011.

[7] Y.K. Panwar, M. Singh, Certain properties of generalized Fibonacci sequence, Turkish J. Anal. Number Theory, 2(1) (2014), 6-8.

[8] G.P.S Rathore, O. Sikhwal, R. Choudhary, Generalized Fibonacci-like sequence and some identities, SCIREA J. Math., 1(1)(2016), 107-118.

[9] O. Sikhwal, Y. Vyas, Generalized Fibonacci-type sequence and its Properties, Int. J. Sci. Res., 5(12) (2016), $2043-2047$.

[10] B. Singh, S. Bhatnagar, Fibonacci-like sequence and its properties, Int. J. Contemp. Math. Sci., 5(18) (2010), 859-868.

[11] B. Singh, S. Bhatnagar, O. Sikhwal, Fibonacci-like sequence, Int. J. Adv. Math. Sci., 1(3)(2013), $145-151$.

[12] M. Singh, Y. Gupta, O. Sikhwal, Identities of generalized Fibonacci-like sequence, Turkish J. Anal. Number Theory, 2(5) (2014), 170-175.

[13] B. Singh, O. Sikhwal, Y. K Gupta, Generalized Fibonacci-Lucas sequence, Turkish J. Anal. Number Theory, 2(6)(2014), 193-197.

[14] N. J. A. Sloane. The On-Line Encyclopedia of Integer Sequences (OEIS), Available at http: / / oe is . org.

[15] A. Wani, G. P. S. Rathore, K. Sisodiya, On the properties of Fibonacci-Like sequence, Int. J. Math. Trends Tech., 29(2) (2016), 80-86. 